\title{
Transtornos Mentais Comuns em Estudantes de Administração de uma Universidade Pública no Agreste Pernambucano
}

\author{
Rayssa Arruda MELO \\ Universidade Federal de Pernambuco (UFPE), Centro Acadêmico do Agreste (CAA), Caruaru, PE, Brasil \\ Elisabeth Cavalcante dos SANTOS \\ Universidade Federal de Pernambuco (UFPE), Centro Acadêmico do Agreste (CAA), Caruaru, PE, Brasil
}

\author{
Pâmela Karolina DIAS
}

Universidade Federal de Pernambuco (UFPE), Centro Acadêmico do Agreste (CAA), Caruaru, PE, Brasil

Received 9 June 20; Accepted 1 August 20.

Evaluation System: Double Blind Review

Editor: Jose Lindenberg Julião Xavier Filho, Dr. ISSN: 2594-8040

To cite this paper: Melo, R. A., Santos, E. C., \& Dias, P. K. (2020). Transtornos mentais comuns em estudantes de administração de uma universidade pública no agreste pernambucano. Journal of Perspectives in Management $J P M, 4$, p. 34-51.

\section{Resumo}

Este trabalho, intitulado Transtornos Mentais Comuns em Estudantes de Administração de uma Universidade Pública no Agreste Pernambucano, tem como objetivo analisar quais os transtornos mentais comuns entre estudantes do curso de Administração do Centro Acadêmico do Agreste, da Universidade Federal de Pernambuco. Para tanto, foi realizada pesquisa quantitativa, exploratória e descritiva com 256 estudantes. Foi realizada identificação sócio demográfica dos sujeitos, mapeamento dos transtornos mentais comuns, fatores que contribuem para os transtornos, e os mecanismos de enfrentamento utilizados. Dentre os resultados encontrados, destaca-se um maior índice de sintomas em estudantes do gênero feminino, fatores motivadores associados ao nervosismo e cobrança sobre si, bem como o uso predominante de instrumentos de defesa focalizados no problema e no suporte social.

Palavras-Chave: Transtorno Mental Comum; Saúde Mental; Curso de Administração. 


\section{Introdução}

Todos os anos muitos estudantes passam pela transição do Ensino Médio para o Ensino Superior, e junto com ela alguns conflitos surgem com frequência (Farias \& Almeida, 2020). Segundo Ferraz e Pereira (2002, p. 150), "a transição para a Universidade coloca em grande destaque os problemas dos alunos, contribuindo para o desenvolvimento de fortes laços com os níveis mais elevados de ansiedade e de stress". Isso acaba sendo um marco significativo por causa das grandes mudanças e adaptações que esses estudantes necessitam enfrentar, gerando conflitos emocionais, insegurança, desorientação, entre tantos outros problemas (Ferraz \& Pereira, 2002; Carlesso, 2020).

A demanda imposta dos professores, a rotina pesada, a privação do lazer, a exigência pela excelência, o sentimento de incapacidade, dificuldade para dormir, a competição predatória no próprio ambiente universitário, incertezas sobre a escolha certa da profissão, as dificuldades no mercado de trabalho, a desmotivação, são algumas das várias inseguranças que assolam a vida de muitos estudantes universitários (Andrade, Sampaio, Farias, Melo, Sousa, Mendonça, Moura Filho, \& Cidrão, 2014; Silva et al., 2019). Percebe-se que muitas instituições têm dado ênfase no desenvolvimento da capacidade técnica e científica na formação de seus alunos, através do desenvolvimento intelectual dos discentes, entretanto, esquece-se de estimular o autoconhecimento, e o relacionamento consigo mesmo e com o próximo, ainda que seu êxito profissional dependa também desse fator (Andrade et al., 2014).

Giglio (1976) afirma que a universidade deve compreender seu papel de estimular e oferecer aos estudantes oportunidades que contribuam com o amadurecimento da personalidade e o desenvolvimento de sua potencialidade, preparando-os para realizar papéis no meio social. O primeiro estudo sobre saúde mental em discentes no Brasil foi realizado pelo professor Galdino Loreto, identificando que, em 1956, cerca de um terço dos discentes atendidos no Serviço de Higiene Mental para estudantes da Universidade Federal de Pernambuco, apresentaram sintomatologia neurótica e dois terços, reações de emoções inadequadas e dificuldades de personalidade. Cerca de uma década e meia após o primeiro estudo, o professor Galdino Loreto realizou uma nova análise que verificou casos com sintomatologia psiquiátrica. Nesse mesmo estudo, 60\% da amostra era de casos sem quadro psiquiátrico definido, que apresentaram "dificuldades emocionais diversas" referentes à fase de formação da "identidade" (Giglio, 1976).

Após esse trabalho, vários outros estudos passaram a ser realizados no Brasil para descrever e compreender melhor esses fenômenos. Cerchiari (2004), por exemplo, identificou que $29 \%$ dos estudantes têm distúrbios psicossomáticos como seu principal problema de saúde mental, seguido de estresse psíquico com $28 \%$, e desconfiança do próprio desempenho que vêm em terceiro lugar com 26\%. Ao estudar a Qualidade de Vida de 558 estudantes de duas universidades públicas brasileiras, a Universidade Estadual de Mato Grosso do Sul (UEMS) e a Universidade Federal de Mato Grosso do Sul (UFMS), Cerchiari (2004) também verificou que transtornos mentais têm maior probabilidade de se desenvolverem no início da vida adulta, especialmente no período universitário, o que também é constatado por Carlesso (2020). Sua amostra foi comparada com estudos de pacientes depressivos em cuidados primários, concluindo que os estudantes universitários têm resultados inferiores ou semelhantes ao grupo de pacientes.

Dados de um levantamento realizado pelo Fórum Nacional de Pró-Reitores de Assuntos Comunitários e Estudantis (FONAPRACE) e pela Associação Nacional dos Dirigentes das Instituições Federais de Ensino Superior (ANDIFES) apontam que 30,45\% dos estudantes graduandos em Universidades Federais já buscaram atendimento psicológico uma vez na vida. Desses, 6,86\% frequentaram o serviço nos últimos 12 meses, 4,73\% estão sob acompanhamento, e $8,9 \%$ já utilizaram algum medicamento psiquiátrico. A pesquisa foi realizada com 939,6 mil alunos de todo o país (FONAPRACE/ANDIFES, 2016).

A partir destas considerações, percebe-se que o estudo sobre a saúde mental de estudantes universitários é um tema importante para a compreensão da dinâmica de organizações de ensino superior. Entender como se sentem os estudantes universitários, e fatores que podem desencadear 
sentimentos de angústia e mal-estar no ambiente acadêmico, pode auxiliar a organização a prevenir problemas futuros, e a lidar com as dificuldades cotidianas dos estudantes.

Observa-se também que estudos que tratam do tema saúde mental em estudantes universitários, tem maior predominância em cursos da área de saúde como medicina, fisioterapia, odontologia, e da área de ciências humanas como a psicologia, com o foco principalmente em ansiedade e depressão (ver Abrão, Coelho, \& Passos, 2008; Alves, 2014; Andrade et al., 2014); Andrade et al., 2016; Leão, Gomes, Ferreira, \& Cavalcanti, 2018; Carlesso, 2020). Diante disso, parece importante pesquisar a saúde mental também em outros cursos.

Nesse sentido, o presente estudo tem como objetivo analisar os transtornos mentais comuns entre estudantes do Curso de Administração do Centro Acadêmico do Agreste (CAA) da Universidade Federal de Pernambuco (UFPE). Para alcançar o objetivo do estudo, foi realizado o mapeamento dos transtornos mais comuns entre os estudantes do curso de Administração do CAA/UFPE, verificaram-se os fatores que podem contribuir para o desenvolvimento de tais transtornos, e foram descritos os principais mecanismos de defesa utilizados pelos estudantes para lidar com tais transtornos

\section{Referencial Teórico}

\subsection{Transtornos mentais no contexto universitário}

Por saúde mental, compreende-se o "estado do mais completo bem-estar físico, mental e social e não apenas a ausência de enfermidade", o que envolve o bem-estar subjetivo, a auto eficácia percebida, a autonomia, a competência, a dependência intergeracional e a auto realização do potencial intelectual e emocional da pessoa (OMS, 2002, p. 30). O Transtorno Mental, por sua vez, é definido pelo Manual Diagnóstico e Estatístico de Transtornos Mentais (DSM-5), como: “[...] perturbação clinicamente significativa na cognição, na regulação emocional ou no comportamento de um indivíduo que reflete uma disfunção nos processos psicológicos, biológicos ou de desenvolvimento subjacentes ao funcionamento mental" (American Psychiatric Association, 2014, p. 20).

O termo "transtornos" ou "distúrbios mentais" é aceito para reportar-se aos problemas psicopatológicos que são diagnosticados pela presença de sintomas, considerados "anormais". Um grupo de sintomas pode ser classificado como uma síndrome, sendo assim, uma determinada síndrome psicológica classificada pode então receber o nome de transtorno mental. Atualmente, reconhecem-se como transtornos mentais os problemas classificados no Manual diagnóstico e estatístico de transtornos mentais (DSM-5), da American Psychiatric Association, e no Código Internacional de Doenças (CID-10) (Assis, 2010).

A Universidade é um ambiente que proporciona os mais diversos desafios, que podem levar ao desenvolvimento de transtornos mentais. Para Xavier, Nunes e Santos (2008), nela são possíveis observar situações que propiciam sofrimento psíquico não só nos discentes, mas também nos profissionais que nela trabalham. Além desses sofrimentos é possível observar também manifestações sintomáticas como absenteísmo, depressão, dependência química, isolamento e fobias.

Cartollo (2013) cita diferentes estressores que os estudantes tendem a enfrentar ao longo de um curso universitário, dentre eles estão a transição do ensino médio para o superior (Farias \& Almeida, 2020), mudança de círculos sociais, novas demandas acadêmicas, dificuldades em relacionar a teoria com a prática profissional, pressões individuais, a carga horária excessiva dos cursos, a realização de provas e as dificuldades enfrentadas em apresentar trabalhos em sala de aula, além de problemas acadêmicos, por não estarem frequentando o curso inicialmente desejado. $\mathrm{O}$ autor ainda afirma que, em muitos casos, a presença desses estressores pode desenvolver impactos negativos na vida do estudante, "comprometendo o seu desempenho acadêmico, promovendo o 
surgimento de distúrbios psicossomáticos e colaborando para o desenvolvimento de comportamentos relacionados à depressão e ao consumo de álcool” (Cartollo, 2013, p. 32).

De acordo com uma pesquisa sobre o perfil socioeconômico e cultural dos estudantes de graduação das instituições federais de ensino superior brasileira, foi constatado que dentre 939.604 estudantes pesquisados, na seção "Dificuldades Estudantis", 21,85\% (205.275) desses estudantes citaram a dificuldade com a adaptação a novas situações como dificuldade que interfere no contexto acadêmico, 16\% (152.424) apresentam dificuldade de aprendizado, 31,14\% (292.576) citaram a carga excessiva de trabalhos estudantis e 19,8\% (186.015) mencionaram a relação professor (a)estudante como a dificuldade que interfere significativamente na vida do discente ou no contexto acadêmico (FONAPRACE/ANDIFES, 2016). Outras dificuldades enfrentadas pelos graduandos, segundo a pesquisa mencionada, são relativas a relacionamentos familiares, sociais e conjugais. Situações de violência psicológica, conflitos de valores/religiosos, discriminação e preconceitos, dificuldade de acesso a materiais e meios acadêmicos, dificuldade financeira, dificuldade de aprendizado, falta de disciplina/hábito de estudo, carga horária de trabalho excessiva, também foram apontados.

Fatores enfrentados pelos alunos no início do curso, como a transição para a nova vida estudantil (Farias \& Almeida, 2020), o grande volume de informações adquiridos rapidamente, a carga horária mais rígida e a responsabilidade que aumenta significativamente devido à escolha de formação de sua carreira, são exemplos de fatores causadores de estresse. A literatura aponta ainda que também é possível observar fatores estressores no final da trajetória acadêmica quando os discentes começam a pensar sobre suas aptidões e mercado de trabalho (Victoria et al., 2013; Aquino, Cardoso, \& Pinho, 2019).

De acordo com Costa e Leal (2008) e Aquino, Cardoso e Pinho (2019), estar cursando uma área de sua preferência ou ter identificação com ela é de suma importância para a adaptação acadêmica, ou seja, alunos que optam pelo curso correspondente à sua primeira opção, tendem a ter sentimentos mais positivos em relação ao curso, assim como melhores perspectivas de carreiras e projetos vocacionais. Ele ainda acrescenta que alunos que frequentam o curso referente à sua primeira opção ou de grande identificação também apresentam um nível de bem-estar psicológico consideravelmente superior em relação àqueles que optaram por escolhas vocacionais secundárias.

A saúde mental dos estudantes também está associada à mudança de casa e de círculo de amigos, isto é, a eventuais perdas de relacionamentos (Silva et al., 2019). Costa e Leal (2008) e Aquino, Cardoso e Pinho (2019) mencionam que estudantes deslocados têm mais problemas de isolamento/solidão e de adaptação à vida acadêmica. Constatam ainda que os estudantes que saem de sua casa para morar mais próximos do campus de estudo apresentam mais ansiedade e maior angústia psicológica, enquanto que os que continuam com seus familiares apresentam melhores resultados, como bem estar físico e psicológico, são mais otimistas, apresentam maior confiança, sendo assim, conseguem uma melhor adaptação acadêmica.

A exemplo da demanda acadêmica, a ansiedade que é gerada nos estudantes no período de provas acaba sendo um importante fator de tensão, e esse problema acaba causando um baixo desempenho acadêmico nas universidades. Segundo Victoria et al. (2013), estudos anteriores comprovam que esta ansiedade é gerada pela preocupação de fracasso nas avaliações. Esse nervosismo provocado nos estudantes pode ser desenvolvido no momento do exame ou até mesmo dias antes da realização da prova. Muitas vezes os discentes estão aptos para realizar a atividade, porém acabam sofrendo as consequências por não conseguirem controlar suas emoções.

Furtado, Falcone e Clark (2003) e Silva et al. (2019) apontam que, em função da necessidade de terem o melhor desempenho escolar, os estudantes se tornam pessoas mais exigentes; são propensos a sofrerem pressões; são pouco tolerantes a falhas; se culpam pelo que não sabem e são paralisados pelo medo de errar. Para as autoras, esse cenário é definido por sentimentos de desvalia e impotência, e acaba sendo responsável por ideias de abandono do curso, depressão e suicídio.

Para Giglio (1976), as dificuldades emocionais dificultam o aprendizado pelo gasto de energia utilizado para tentar solucionar os conflitos. Ele verificou que o rendimento acadêmico 
normalmente ocupa um alto posto no que dizem respeito a valores universitários, deste modo, as falhas costumam dar lugar a reações depressivas, que dependendo da personalidade do universitário pode lhe afetar com maior ou menor intensidade. O mesmo autor, citando Murguia (1971), afirma que "o abandono dos estudos é, com bastante frequência, mais consequência de distúrbio emocional do que de dificuldades intelectual" (Giglio, 1976, p. 13).

Giglio (1976), citando um estudo de Nicholi (1967), diz que este autor, ao relacionar o abandono da faculdade com problemas de saúde mental, constatou que os alunos da Universidade de Harvard que tinham uma maior tendência ao abandono do curso, eram aqueles que apresentavam um maior "desempenho intelectual". O autor conclui afirmando que esse fato acaba indo de encontro à crença de que os dotes intelectuais são responsáveis pela permanência dos estudantes na universidade.

A evasão universitária pode ser definida como o "desligamento do curso superior por qualquer outro motivo que não a diplomação" (Castro \& Teixeira, 2014, p. 10). Estudos indicam uma média de $12 \%$ na evasão das universidades públicas, além de afirmar que o primeiro ano de curso é o período mais propenso para que o aluno opte por desistir, pois é quando este ainda está em momento de transição, e que seu sucesso ou fracasso são os verdadeiros determinantes da desistência (Andrade et al., 2016).

O uso de drogas, sejam elas lícitas ou ilícitas, podem passar a fazer parte do cotidiano do estudante como uma forma que este tem de enfrentar diversas situações estressantes que a universidade proporciona. De acordo com o I Levantamento Nacional sobre o uso de Álcool, Tabaco, e outras Drogas entre Universitários das 27 Capitais Brasileiras, publicado em 2010 pela Secretaria Nacional de Políticas sobre Drogas (SENAD), no ano de 2009, 49\% dos estudantes pelo menos uma vez na vida já experimentaram alguma droga ilícita, e $80 \%$ dos que se declararam menores de 18 anos já consumiram alguma bebida alcoólica. A pesquisa constata também que o consumo de álcool, tabaco e outras drogas são mais frequentes entre os universitários do que na população em geral. É que comparando os gêneros, os homens usam mais maconha, inalantes, cloridrato de cocaína, alucinógenos, ecstasy e esteroide. Já as mulheres consomem mais anfetamínicos, tranquilizantes e analgésicos opiáceos (Andrade, Duarte, \& Oliveira, 2010; Carlesso, 2020).

É importante ressaltar que estudos apontam que existe diferença em relação ao gênero no que se refere à saúde mental. As mulheres estão mais propensas a terem depressão e ansiedade, assim como são a maioria entre os que procuram ajuda profissional e também entre os respondentes (ver Nogueira \& Sequeira, 2017; Leão et al., 2018; Aquino, Cardoso, \& Pinho, 2019; Silva et al., 2019).

Carlotto (2013) afirma que embora em sua pesquisa não tenha encontrado ligação entre adaptação acadêmica e o uso de substâncias psicoativo, o uso do álcool, por exemplo, está associado a problemas acadêmicos como a não realização de trabalhos acadêmicos e de provas, absenteísmo e interrupção dos estudos. Além de interferir nas atividades acadêmicas, esse hábito pode afetar também a atuação profissional desses jovens.

\subsection{Transtornos mentais comuns e mecanismos de enfrentamento}

Em pesquisa do Fonaprace/Andifes (2016), na seção "Dificuldades Estudantis", os estudantes foram questionados sobre os tipos de dificuldades emocionais que podem ter interferido na sua vida acadêmica nos últimos 12 meses. A ansiedade ficou em primeiro lugar na pesquisa, com 58,36\% (548.372), seguido de desânimo/falta de vontade de fazer as coisas com 44,72\% (420.202). Insônia ou alteração significativa de sono apareceu em terceiro lugar com 32,57 (306.038), seguido de sensação de desamparo/desespero/desesperança com 22,55\% (211,869), sentimento de solidão com 21,29\% (200.057), sensação de desatenção/ desorientação/ confusão mental com 19,3\% (181.336), tristeza persistente com 19,28 (181.142), medo/pânico com 10.56\% (99.213), pensamento suicida $4,13 \%$ (38.838). 
Os transtornos de ansiedade segundo o DSM-5

Incluem transtornos que compartilham características de medo e ansiedade excessivos e perturbações comportamentais relacionados. O medo é a resposta emocional a ameaça iminente real ou percebida, enquanto ansiedade é a antecipação de ameaça futura.[...] esses dois estados se sobrepõem, [...] o medo sendo com mais frequência associado a períodos de excitabilidade autonômica aumentada, necessária para luta ou fuga, pensamentos de perigo imediato e comportamentos de fuga, e a ansiedade sendo mais frequentemente associada a tensão muscular e vigilância em preparação para perigo futuro e comportamentos de cautela ou esquiva (American Psychiatric Association, 2014, p. 189).

Ainda, segundo o manual, os transtornos de ansiedade diferem entre si, podendo se configurar como transtorno de ansiedade de separação, mutismo seletivo, fobia específica, transtorno de ansiedade social (fobia social), transtorno de pânico, transtorno de ansiedade generalizada, e ansiedade induzida por substância/medicamento (American Psychiatric Association ,2014).

Os transtornos depressivos segundo o DSM-5 (Manual diagnóstico e estatístico de transtornos mentais) têm como característica "a presença de humor triste, vazio ou irritável, acompanhado de alterações somáticas e cognitivas que afetam significativamente a capacidade de funcionamento do indivíduo" (American Psychiatric Association, 2014, p. 155). Ainda segundo o manual, o Transtorno Depressivo Maior representa a condição clássica desse grupo de transtornos e é caracterizado por episódios distintos de pelo menos duas semanas de duração, envolvendo alterações nítidas no afeto (sente-se deprimido, vazio, sem esperança), na cognição (capacidade diminuída para pensar ou se concentrar, ou indecisão) e em funções neurovegetativas, (perda ou ganho significativo de peso sem estar fazendo dieta, ou redução ou aumento do apetite) e remissões interepisódicas.

Fonseca, Guimarães e Vasconcelos (2008) denominam "Sofrimento Difuso" os sintomas inespecíficos, ou queixas somáticas, tais como cefaleia, fadiga, insônia, nervosismo e estado de mal-estar que não são classificáveis nos diagnósticos médicos ou psiquiátricos. Sendo assim, Transtornos Mentais Comuns acaba sendo uma classificação possível para o diagnóstico. Esses transtornos podem se apresentar através de múltiplos sintomas, como por exemplo, queixas somáticas inespecíficas, irritabilidade, esquecimento, falta de concentração, assim como várias manifestações que possam facilmente se caracterizar como sintomas depressivos, ansiosa ou somatoformes.

Estudos sobre a saúde mental dos estudantes tem sido realizados em diversos países (Cunha et al., 2009). Com isso têm surgido também os mais variados conceitos para caracterizar os distúrbios não psicóticos, referindo-se em particular às distinções entre depressão e ansiedade. Exemplo disso são os estudos designados por Morbidade Psiquiátrica Menor (MPM), Transtornos Mentais Comuns (TMC) e Problemas Psiquiátricos Menores (PPM) (Cerchiari, 2004).

Para Andrade et al. (2016, p. 833), distúrbio psiquiátrico menor é caracterizado como o "conjuntos de manifestações de mal-estar psíquico, de caráter inespecífico, com repercussões fisiológicas e psicológicas que podem gerar limitações", e é comum identificar entre os estudantes manifestações sintomáticas de transtornos mentais comuns (TMC). Independente de ter recebido um diagnóstico psiquiátrico formal, o transtorno causa sofrimento psíquico, impacto nos relacionamentos e na qualidade de vida, comprometendo, desse modo, o desempenho nas atividades do cotidiano, sendo um fator propulsor para o desenvolvimento de transtornos mais graves (Fiorotti et al., 2010).

A cefaleia, por exemplo, é um dos sintomas que compõem esses quadros de TMC (Silva et al., 2019). Existem vários tipos de cefaleia crônica diária, a exemplo da tensional, da enxaqueca entre outros termos. Uma pesquisa realizada com o intuito de investigar a prevalência de cefaleia entre estudantes de Medicina e Psicologia constatou que $98 \%$ dos alunos sofreram de cefaleia pelo menos uma vez na vida, e $91 \%$ no ano anterior da pesquisa. Dentre os estudantes de medicina, os que tiveram cefaleia tensional corresponderam a 59\%, e enxaqueca, 22\%. Nos estudantes de psicologia, $48,5 \%$ e $32 \%$ respectivamente. Eles continuam afirmando que de todos os estudantes, 
$45 \%$ sofreram interferência variável na produtividade, $77 \%$ se automedicaram e $36 \%$ perceberam piora da cefaleia em relação ao início do curso (Ferri-de-Barros et al., 2011 citado por Andrade et al., 2016).

Os distúrbios de sono são mais um transtorno que os estudantes universitários podem enfrentar. Normalmente a vida dos estudantes universitários é caracterizada por padrão de sono irregular, definido por atrasos no início e final do sono, existindo, normalmente, curta duração de sono no decorrer da semana, e nos finais de semana o sono é substituído por períodos mais prolongados (Almondes \& Araújo, 2003).

Estudos têm encontrado correlação dos distúrbios do sono com problemas de saúde, rendimento diário e bem-estar. Muller e Guimarães (2007), Leão et al. (2018) e Aquino, Cardoso, e Pinho (2019) afirmam que são várias as consequências que a irregularidade do sono pode provocar, sendo alguns deles: a irritabilidade, falta de atenção, aumento no risco de sofrer um acidente ou de um derrame cerebral, enfraquecimento do sistema imunológico, emoções afetadas provocando uma instabilidade emocional, problemas de concentração e memória, assim como dores de cabeça, medo, tensão, fadiga, entre tanto outros males.

Almondes e Araujo (2003) relatam que os estudantes italianos durante a semana dormiam menos que o normal, e reclamavam de sonolência diurna e cochilos mais frequentemente que a população em geral. As consequências dessa irregularidade eram baixo desempenho acadêmico, sintomas de ansiedade e depressão, além de maior uso de tabaco, álcool e cafeína. Os autores continuam afirmando que muitos estudantes entram em situação de conflito quando tem que escolher entre suprir a necessidade de sono e atender às demandas acadêmicas, e que essa situação pode provocar alterações no estado afetivo.

Ainda segundo o DSM-5, os indivíduos com Transtornos do sono-vigília geralmente apresentam:

queixas de insatisfação envolvendo a qualidade, o tempo e a quantidade de sono. [...] Com frequência, os transtornos do sono são acompanhados de depressão, ansiedade e alterações cognitivas, que deverão ser incluídos no planejamento e no gerenciamento do tratamento. Além disso, os distúrbios persistentes do sono (tanto insônia como sonolência excessiva) são fatores de risco estabelecidos para o desenvolvimento subsequente de doenças mentais e de transtornos causados pelo uso de substâncias (American Psychiatric Association, 2014, p. 361).

Outro fator que afeta a vida acadêmica dos estudantes é o estresse. Furtado, Falcone e Clark (2003) dizem que esse termo pode ser entendido como uma reação entre componentes físicos e/ou psicológicos do organismo. Essas reações são resultantes das alterações psicofisiológicas que ocorrem no indivíduo quando este enfrenta situações que ocasionam irritação, medo, excitação ou confusão, ou até mesmo uma felicidade exacerbada.

Diante desses sintomas, os universitários comumente utilizam estratégias de enfrentamento, ou coping - como são denominadas no âmbito internacional. O coping é entendido como esforços cognitivos e comportamentais que os indivíduos utilizam para lidar com situações de dano ou ameaça normalmente decorrentes de situações de estresse. Em outros termos, ao vivenciar situações de estresse, as pessoas tendem a experimentar sensações emocionais desagraváveis, e como resposta, os indivíduos tendem a adotar estratégias para minimizar o sofrimento (Carlotto, 2013).

Como forma de classificar o coping, inúmeras propostas têm sido utilizadas para sua sistematização, e suas duas categorias principais são baseadas na função dos esforços realizados e outra na intenção. O coping focado na emoção utiliza seus esforços para regular o estado emocional, ao invés de solucionar o problema que gera o estresse. Quanto ao segundo tipo, o coping focado no problema, utiliza estratégias para atuar na origem do estresse, e assim tentar modificá-lo. Este é baseado no confronto e na resolução de problema (Carlotto, 2013). Além dessas, outras duas propostas têm sido utilizadas para a classificação do coping, com intuito de promover um maior nível de detalhamento: o coping focado nas relações interpessoais, no qual as estratégias são 
vinculadas à busca de suporte social, como família e amigos; e o coping religioso, que são as crenças e comportamentos religiosos e espirituais utilizados pelos estudantes diante de situações estressoras (Carlotto, 2013).

Zonta, Robles e Grosseman (2006) descrevem diversos caminhos que podem ser utilizados para promover a qualidade de vida de estudantes de medicina. Dentre as estratégias apontadas estão a valorização dos relacionamentos interpessoais (com parentes, namorados, amigos e colegas); a busca de maior equilíbrio entre estudo e lazer; organização do tempo; prática atividade física; cuidados com a saúde, alimentação e sono; ter atividades religiosas ou espirituais; trabalhar a própria personalidade; utilizar técnicas de relaxamento ou meditação e a procura por assistência psicológica. Os autores ainda salientam a importância da criação de serviços de assistência psicológica aos estudantes.

Cunha et al. (2009) afirmam que é importante que exista nas instituições um programa formal que disponibilize aos discentes acompanhamento psicológico, como parte de um plano de atenção a saúde dos estudantes, para que eles consigam lidar com as dificuldades geradas em sua graduação. Os programas de assistência estudantil são uma boa opção e, de acordo com Assis (2010), ofertar serviço de assistência estudantil significa cuidar dos aspectos pessoais da vida de estudantes, refletindo o compromisso que a universidade tem com a comunidade e com a sociedade que a mantém. Ressalta-se também a importância de promover políticas públicas que garantam o acesso e a permanência dos estudantes universitários, como reforçam Barreto, Bezerra e Barbosa (2005).

\section{Procedimentos Metodológicos}

No que diz respeito à natureza do estudo, este é de caráter quantitativo, uma vez que envolve elementos como a objetividade, a predição e o controle (Creswell, 2010). No que se refere aos objetivos, a pesquisa é exploratória e descritiva. Os sujeitos dessa pesquisa foram os estudantes do curso de Administração do Campus Acadêmico do Agreste - CAA da Universidade Federal de Pernambuco - UFPE, sendo o único critério de seleção que esses estudantes estivessem matriculados, independente do período $\left(1^{\circ}\right.$ ao $\left.9^{\circ}\right)$ ou turno (manhã e noite) do curso. Pensando nisso, no questionário foi perguntado qual o período que o sujeito estava matriculado a fim de identificar respondentes que fugissem a esse critério.

O CAA foi o primeiro campus da UFPE no interior de Pernambuco, inaugurado em março de 2006, com o objetivo de contribuir com o desenvolvimento social, econômico e cultural da região e do Estado. A partir de cálculo de intervalo de confiança de $95 \%$, com margem de erro de $5 \%$, chegou-se no número de 252 alunos necessário para compor a amostra. Desse modo, dos 726 alunos regularmente matriculados no curso de administração, na época de confecção dessa pesquisa (2018.1), 256 responderam o questionário.

O questionário foi elaborado no Google Forms para a coleta de dados on-line e continha sessenta questões e estas foram divididas em quatro blocos. É importante dizer que o questionário foi divulgado em grupos das turmas de administração na plataforma do Whatsapp, e Facebook. O primeiro bloco do questionário solicitou dados sociodemográficos dos estudantes; o segundo bloco mapeou os transtornos com base em alguns dos sintomas que são descritos no Manual DSM-5 (American Psychiatric Association, 2014); o terceiro bloco foi sobre os fatores que podem contribuir para o desenvolvimento de transtornos mentais, com questões adaptadas do questionário de Vivências Acadêmicas (QVA) (Almeida \& Ferreira, 1997); o último bloco abordou os mecanismos de enfrentamento, adaptando questões do questionário "Escala Modos de Enfrentamento de Problemas (EMEP)" traduzido por Gimenes e Queiroz (1997) e adaptado para a população brasileira por Seidl, Troccoli e Zannon (2001).

Os dados foram tratados utilizando estatística descritiva, por meio de cálculo de porcentagens e médias. A seção de apresentação e discussão dos resultados foi dividida em quatro partes: dados sócios demográficos, mapeamento dos transtornos, situações que contribuem para os transtornos, e mecanismos de enfrentamento. 


\section{Apresentação e Discussão dos Resultados}

\subsection{Dados Sociodemográficos}

Dentre os estudantes que participaram da pesquisa, a amostra indicou que $139(54,3 \%)$ dos respondentes são do gênero feminino e 117 (45,7\%) são do masculino, 178 (68,5\%) tem faixa etária entre 19 e 25 anos, e $124(48,4)$ se declararam pardos. Esse resultado vai ao encontro do que diz a literatura, que indica que as mulheres em pesquisas sobre esta temática são a maioria entre os respondentes (Nogueira \& Sequeira, 2017; Leão et al., 2018; Aquino, Cardoso \& Pinho, 2019).

Quando questionados sobre a moradia, 196 estudantes afirmaram que não precisaram mudar por causa da graduação, e isso equivale a cerca de $76,6 \%$ do total de alunos, enquanto 60 deles mudaram. Consideramos este um dado importante, pois, como afirma Cartollo (2013) e Silva et al. (2019), a mudança de cidade pode ser um fator estressor para o jovem universitário, uma vez que essa mudança pode ser associada a possíveis perdas de relacionamentos.

Costa e Leal (2008) declaram que estudantes que mudam de cidade, para ficar mais próximo do campus universitário, têm mais problemas de isolamento e solidão, além de apresentarem mais ansiedade e uma maior angústia psicológica. Ainda sobre a moradia, 118 estudantes residem em Caruaru, 22 em Bezerros, 19 em Belo Jardim, 11 em Gravatá, 8 em Santa Cruz do Capibaribe, e cerca de 6 em outras cidades do entorno.

A grande maioria dos alunos afirmou também que atualmente reside com os pais $(65,2 \%)$. Nos itens que se referem ao trabalho e a renda mensal bruta familiar, 134 (52,3\%) alunos afirmaram possuir um trabalho remunerado e $92(35,9 \%)$ tem renda familiar de 1 salário mínimo e até 2 salários mínimos. Ao serem perguntados sobre o período e o turno do curso que estão matriculados atualmente, 27 estudantes indicaram estar no $9^{\circ}$ período/noite; 16 no $2^{\circ}$ período/manhã; 14 no $3^{\circ}$ período/manhã; 14 no $7^{\circ}$ período/noite; 13 no $4^{\circ}$ período/noite; 13 no $8^{\circ}$ período/noite. Os demais períodos tiveram uma quantidade inferior a 11 alunos.

Ademais, cerca de um quarto do total de estudantes afirmaram ter se privado de alguma atividade de lazer após o ingresso a universidade. Esse número equivale a 79,3\% do total, e se mostra preocupante, dada a importância das atividades físicas e de lazer para uma boa saúde física e mental, como afirmam autores como Xavier, Nunes e Santos (2008), Furtado, Falcone e Clark (2003), Zonta, Robles e Grosseman (2006) e Silva et al. (2019).

\subsection{Mapeamentos dos Transtornos}

Foi observado que apenas $33,2 \%$ dos estudantes já procuraram atendimento psicológico alguma vez na vida, e que apenas 7,4\% dos entrevistados possuem acompanhamento psicológico atualmente, indicando que a maioria dos estudantes não tem um acompanhamento de sua saúde mental. Além disso, dos estudantes respondentes, apenas $9 \%$ já foram diagnosticados com algum tipo de transtorno.

Ao realizar recorte de gênero, percebe-se que, dos estudantes que afirmaram já ter procurado algum atendimento psicológico, o gênero feminino foi o que teve maior porcentagem, sendo esta de $58,8 \%$, enquanto o gênero masculino representa $41,2 \%$ do total. Quando perguntado se o estudante está sendo acompanhado por algum profissional da área de psicologia atualmente, 63,2\% das mulheres afirmaram que sim, indicando mais uma vez a predominância feminina. Os resultados aqui encontrados conversam com Silva et al. (2019) que afirmam que as mulheres são as que mais buscam apoio profissional. Entre os homens, essa porcentagem foi de 36,8\%. Por fim, quando questionados se já foram diagnosticados com algum transtorno mental 52,2\% das mulheres afirmaram que sim, contra 47,8\% dos homens. Esse achado corrobora com outros estudos que afirmam que mulheres tem uma inclinação maior a ter depressão e ansiedade, por exemplo (Nogueira \& Sequeira, 2017; Leão et al., 2018).

Após a identificação dessas informações mais gerais, buscou-se levantar informações referentes aos Transtornos Mentais Comuns ou Sofrimento Difuso que, de acordo com Fonseca, 
Guimarães e Vasconcelos (2008), refere-se a sintomas inespecíficos ou queixas somáticas. A maioria dos estudantes disse que não sente dor de cabeça frequentemente (59\%), apesar do número de pessoas que sente ser alto (49\%). Algo similar acontece com a dificuldade de pensar com clareza frequentemente: a maioria dos estudantes diz não ter dificuldade $(51,2 \%)$, mas uma quantidade considerável possui essa dificuldade $(48,8 \%)$.

A maioria dos respondentes não sente falta de apetite frequentemente $(73 \%)$ nem tem chorado mais que o de costume $(71 \%)$. Mas a maioria tem tido insônia $(57,4 \%)$, dificuldade de tomar decisões $(54,7 \%)$, dificuldade de realizar com satisfação suas atividades diárias $(74,6 \%)$, dificuldade de lembrar-se das coisas $(61,7 \%)$, tem se sentido cansado o tempo todo $(77 \%)$, tem tido alterações de humor constantes $(61,7 \%)$, e tem se sentido triste ultimamente (59\%).

Como se percebe acima, o item "atualmente me sinto cansado o tempo todo" foi o mais citado pelos estudantes, com um total de 197 concordâncias, o que equivale a $77 \%$ do total de alunos entrevistados. Quando esses valores são separados por gênero, é possível perceber que as mulheres são as que mais sofrem com esse problema com uma porcentagem de 58,9\%, enquanto os homens representam 41,1\% dos que sofrem desse sintoma. Ainda sobre esse item, percebeu-se que, entre os estudantes que se queixam de cansaço constante, $152(77,2 \%)$ são aqueles que não residem na cidade onde o campus é localizado e precisam realizar viagens diárias para estudar.

A dificuldade em realizar atividades diárias com satisfação foi citada pelos estudantes como sendo a segunda maior dificuldade que eles têm enfrentado ultimamente, depois de sentir-se cansado o tempo todo. A partir do levantamento realizado, 55\% das mulheres concordam que sentem dificuldades para realizar com satisfação suas atividades diárias, enquanto a porcentagem para os homens é um pouco inferior com $45 \%$ do total. Esse fator também apareceu na pesquisa do Fonaprace/Andifes (2016) como a segunda dificuldade mais enfrentada pelos estudantes universitários entrevistados. A alteração de humor constante foi outro fator muito citado pelos alunos, sendo o terceiro problema que os alunos do curso de administração do CAA enfrentam constantemente. Do total de $61,7 \%$ dos entrevistados que responderam sim à questão, 58,9\% dos estudantes foi do gênero feminino e $41,1 \%$ do gênero masculino.

Assim como alteração de humor, ter dificuldade de lembrar as coisas foi o terceiro motivo que os estudantes afirmam ter que enfrentar constantemente. Nesta questão, assim como nas demais, a porcentagem doo gênero feminino foi maior que a do gênero masculino, com um total de $57 \%$ e $43 \%$ respectivamente. Em relação a essa questão, a literatura aponta que essa falha na memória está associada à ansiedade, pois pessoas com esse transtorno tendem a querer antecipar ameaças futuras, gerando assim pensamentos acelerados e algumas vezes desordenados, características que podem provocar no indivíduo um desvio no foco da memória.

Sobre a presença de cefaleia, um recorte de gênero evidencia que, do total de estudantes que afirmam sentir dores de cabeça frequentemente, $61 \%$ é do gênero feminino e $39 \%$ do gênero masculino. A cefaleia, em seus mais variáveis tipos, é um dos sintomas característicos de TMC. Andrade et al. (2016), realizaram uma pesquisa que constatou que devido à cefaleia, parte dos estudantes entrevistados sofreram interferência variável na produtividade, se automedicaram e perceberam piora da cefaleia em relação ao início do curso.

Sobre a frequente falta de apetite, o gênero feminino foi o que mais concordou com a afirmação, representando $62,3 \%$ do total, enquanto no gênero masculino essa porcentagem é de 37,7\%. Na pesquisa realizada pela FONAPRACE/ANDIFES (2016), para saber o perfil socioeconômico e cultural dos estudantes de graduação das instituições federais de ensino superior brasileira, "problemas alimentares" acabou sendo um dos fatores citados pelos estudantes na seção "Dificuldades Estudantis", no qual cerca de $13 \%$ dos estudantes respondeu que passou por problemas alimentares, podendo ser problemas como alterações de peso ou de apetite, bulimia ou anorexia (FONAPRACE/ANDIFES, 2016). Em relação ao sintoma "dificuldade contínua de pensar com clareza", as mulheres foram as que mais concordaram com a afirmação com uma porcentagem de $54,4 \%$, enquanto nos homens essa porcentagem foi de $45,6 \%$.

Quando questionados se sentem insônia ou alterações significativas de sono constantemente, 147 dos alunos afirmaram que sim. Desses estudantes, cerca de 58,5\% são do gênero feminino, 
enquanto 41,5\% do gênero masculino. Muller e Guimarães (2007), Leão et al. (2018) e Aquino, Cardoso, e Pinho (2019) afirmam que as pessoas tendem a enfrentar várias consequências devido à irregularidade do sono, tais como: irritabilidade, medo, instabilidade emocional, aumento no risco de sofrer um acidente ou de um derrame cerebral, problemas de concentração e memória, dores de cabeça, tensão, fadiga, entre tanto outros males. No item que pergunta sobre a dificuldade que os alunos têm de tomar decisões, mais uma vez o gênero feminino foi o que mais concordou com a questão com 60,7\% do total de alunos, enquanto o gênero masculino ficou com 39,3\%.

Quando os estudantes foram questionados se tem se sentido tristes ultimamente, 58,3\% das mulheres concordaram com a afirmação, e a porcentagem para os homens foi de $41,7 \%$. Na pesquisa da FONAPRACE/ANDIFES (2016), "tristeza persistente" também foi um fator citado na seção onde são apresentadas as principais dificuldades pelas quais podem passar os estudantes de graduação. Quando a pergunta foi se os estudantes estão chorando mais do que de costume, o gênero feminino foi o que apresentou uma maior porcentagem, com 69,9\%. O número no gênero masculino é menor, com $30,1 \%$.

Diante dos sintomas evidenciados aqui, foi feito também um levantamento dos alunos que têm probabilidade de desenvolver transtorno depressivo maior, o qual é considerado representante clássico dos transtornos depressivos, segundo o DSM-5 (Manual diagnóstico e estatístico de transtornos mentais). As características desse grupo são alterações nítidas no afeto, sentir-se deprimida, vazia, sem esperança; na cognição, sua capacidade é diminuída para pensar ou se concentrar, evidenciando indecisão; e em funções neurovegetativas, tem perda ou ganho significativo de peso sem estar fazendo dieta, ou redução ou aumento do apetite (American Psychiatric Association, 2014).

Para fazer o levantamento, foram selecionados os estudantes que responderam "sim" em todas as questões que compuseram o segundo bloco de questões, ou seja, aqueles estudantes que afirmaram sentir todos os sintomas tidos como Transtornos Mentais Comuns. Chegou-se à conclusão de que o total de estudantes que apresentam características que possibilitam o desenvolvimento do Transtorno Depressivo Maior (TDM) é de 13 alunos respondentes, sendo os estudantes do gênero feminino $92,3 \%$ do total.

Percebe-se também que dentre os alunos que apresentaram características suscetíveis ao desenvolvimento de transtornos depressivo maior (TDM), 27,3\% são os estudantes que precisaram mudar de cidade devido o ingresso à universidade, isso equivale a cerca de um quarto do totalQuando as mudanças são acompanhadas de experiências perturbadoras, elas trazem consigo consequências na saúde física e psíquica do indivíduo (Costa \& Leal, 2008; Silva et al., 2019).

\subsection{Fatores que contribuem para os transtornos}

Sobre os fatores considerados, foi possível perceber que a maioria dos estudantes discorda total ou parcialmente sobre ter dificuldades de fazer novas amizades, sobre ter dificuldade de relacionamento com professores, com técnicos ou com terceirizados. Desse modo, o relacionamento entre aluno e pessoas da universidade não se apresenta como um ponto problemático para os estudantes questionados. Nos itens que versam sobre a disponibilidade dos professores em atender e orientar os alunos, e sobre a relação de respeito e ética com o aluno, a maioria dos estudantes respondeu que concorda parcialmente com a afirmação, mostrando assim que os alunos consideram esses itens como adequados.

Nos itens relacionados às cobranças dos alunos feitas a si mesmo e ao nervosismo que é gerado por meio das atividades acadêmicas, os alunos demonstraram, em sua maioria, indiferença, concordância parcial e total com as afirmativas, respectivamente. Esse resultado corrobora com o estudo de Cartollo (2013), que menciona que a pressão individual e as dificuldades em realizar atividades acadêmicas são fatores estressores que o estudante tende a enfrentar ao longo de sua formação acadêmica. Ele afirma que a presença desses fatores pode provocar no indivíduo impactos 
negativos, como por exemplo, o comprometimento do desempenho acadêmico, ou até mesmo o desenvolvimento de distúrbios psicossomáticos.

A maioria dos estudantes concordam totalmente com o item "Sou exigente comigo mesmo, para ter um ótimo desempenho acadêmico", o que também aconteceu com os itens "Fico nervoso (a), tenso (a) ou preocupado (a) para realizar alguma atividade acadêmica (inclui provas, trabalhos ou seminários)" e "o nervosismo, tensão ou preocupação já foram motivos que me levaram a ter um baixo desempenho acadêmico (inclui provas, trabalhos ou seminários)". Esses resultados corroboram com estudo realizado por Victoria et al. (2013), pois nele é comprovado que a ansiedade é gerada nos estudantes devido à preocupação de fracasso nas avaliações, e que essa ansiedade pode ser desenvolvida dias antes ou durante a realização das atividades.

A maioria dos estudantes concorda parcialmente com a afirmação "Sinto-me mal e tenho dificuldade de me perdoar quando tenho um baixo desempenho nas avaliações acadêmicas (inclui provas, trabalhos ou seminários)", correspondendo a $73(28,5 \%)$, e boa parte deles $(23,8 \%)$ concorda totalmente com a afirmativa. Esse resultado pode ser explicado por Furtado, Falcone e Clark (2003), quando mostram que, com o intuito de terem o melhor desempenho acadêmico, os discentes se tornam mais exigentes consigo mesmo e com isso ficam menos tolerantes ao erro. Outra observação que as autoras fazem é que essas atitudes geram no estudante um sentimento de desvalia, sendo um dos possíveis causadores de abandono do curso. Giglio (1976) diz que o rendimento acadêmico é considerado um dos valores universitários, e que a imperfeição nesse quesito pode causar no aluno reações depressivas, as quais podem ser de maior ou menor intensidade.

Com base nos dados coletados no item da pesquisa que fala sobre a dificuldade que o(a) aluno(a) tem de se perdoar devido um baixo desempenho nas avaliações acadêmicas, foi feito um gráfico com o objetivo de saber a porcentagem de estudantes que já pensaram em trancar ou desistir do curso.

Como é possível perceber no gráfico 1 abaixo, dentre os estudantes que concordam totalmente em ter dificuldade de se perdoar devido ao baixo desempenho acadêmico, foi maior o número de alunos que discordaram totalmente da afirmativa "já pensei em trancar ou desistir do curso", sendo este número equivalente a 20. Entretanto, se analisarmos os dados, a diferença entre o número de alunos que em algum momento já pensou em trancar ou desistir do curso, e aqueles que discordam totalmente da afirmativa. é mínima, o que faz dessa afirmação algo preocupante, uma vez que Giglio (1976) menciona que a desistência dos estudos está mais relacionada com distúrbios emocionais do que por dificuldade intelectual do indivíduo.

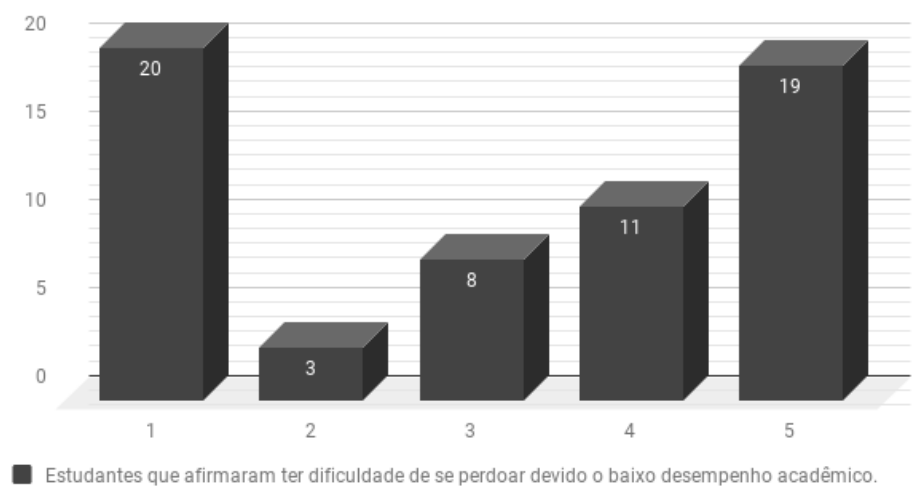

Gráfico 1 - Alunos que concordam totalmente em ter dificuldade de se perdoar devido ao baixo desempenho acadêmico, e que já pensaram em trancar ou desistir do curso.

Fonte: Elaboração Própria, 2020.

Os itens do questionário relativos aos métodos de ensino utilizados pelos professores para alcançar os objetivos do curso, e a adequação entre as tarefas exigidas e o tempo de sua realização, demonstrou indiferença por parte dos estudantes respondentes. Já quando são perguntados se o conteúdo ministrado em sala de aula supre as necessidades do mercado de trabalho, a maior parte 
dos alunos discorda parcialmente desta afirmação, o que pode ser um fator estressor para o estudante que está próximo do término do curso. Os últimos itens, referentes à troca e ao trancamento do curso, tiveram maior quantidade de resposta "discordo totalmente" com uma quantidade de $99(38,7 \%)$ e $86(33,6 \%)$ respectivamente.

Para o item referente ao curso de administração como primeira opção de curso, foi constatado que 46 do total de alunos entrevistados na pesquisa discordam totalmente que o curso de Administração corresponde a sua primeira opção de curso, enquanto 92 alunos afirmam concordar totalmente com a afirmação. Isso corresponde a uma porcentagem de $18 \%$ e $35,9 \%$ respectivamente. Através dessas afirmações, foi desenvolvido um gráfico (gráfico 2), no qual é possível perceber que dos 92 alunos que declaram administração como sendo a sua primeira opção de curso, 68 concordam que estão totalmente satisfeitos com o curso, e apenas 2 pessoas discordam totalmente com a afirmação.

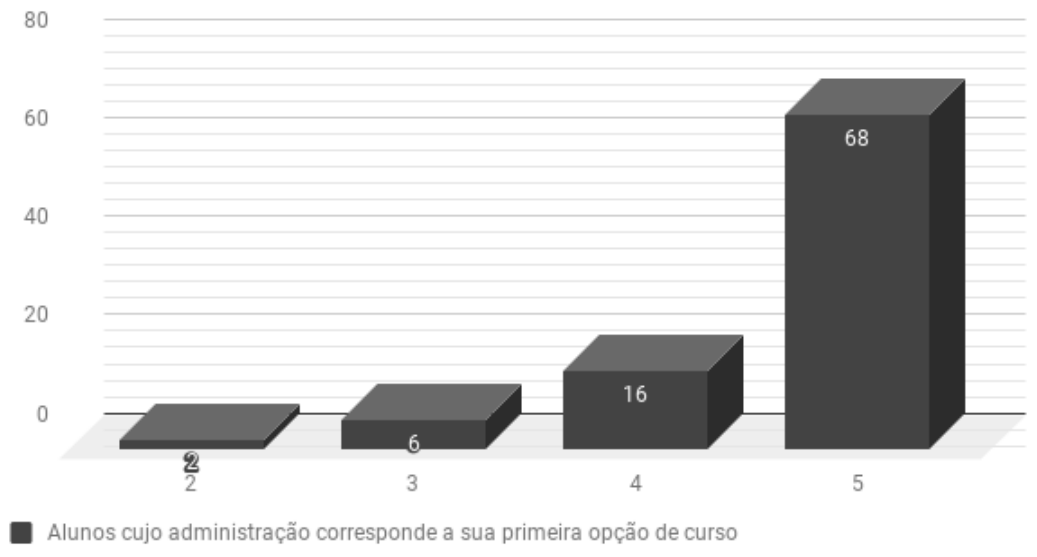

Gráfico 2 - Satisfação do aluno com a escolha do curso, quando o mesmo corresponde a sua primeira opção.

Fonte: Elaboração Própria (2019).

Por meio dessas informações, podemos perceber que a maioria dos alunos que dizem estarem satisfeitos com o curso de administração são aqueles que tinham o curso como sua primeira opção, certificando assim o que já foi percebido por Costa e Leal (2008) e Aquino, Cardoso e Pinho (2019), que afirmam que esse fator é de suma importância para adaptação acadêmica do aluno, pois por meio dele os estudantes se sentem mais positivos com o curso, podendo apresentar também um melhor nível de bem-estar psicológico.

\subsection{Mecanismos de enfrentamento}

Como já foi apresentado no referencial teórico, mecanismos de enfrentamento, ou coping, são métodos utilizados pelo indivíduo para lidar com situações adversas, ou seja, são as estratégias de enfrentamento utilizadas para minimizar o possível sofrimento (Carlotto, 2013). Nos itens do questionário relacionados ao uso de substâncias lícitas e ilícitas (drogas, medicação, álcool) os respondentes afirmaram não fazerem uso, dado que a maioria respondeu "discordo totalmente" quando questionados sobre a utilização desses recursos.

Dentre essas perguntas foi percebido que 20 alunos declararam concordar totalmente com a alternativa que fala sobre o início do uso de substâncias psicoativas após o ingresso na universidade, o que equivale a $7,8 \%$ do total de alunos entrevistados. Um número considerado pequeno, mas uma informação que já tinha sido percebida por estudos anteriores, a exemplo do estudo que foi publicado pela Secretaria Nacional de Políticas sobre Drogas (SENAD), que declara que o início da formação acadêmica pode constituir, para alguns jovens, um momento de maior vulnerabilidade, fazendo com que eles fiquem mais suscetíveis ao uso de drogas (Andrade, Duarte, \& Oliveira, 2010). 
No item "Quando passo por algum problema na universidade, eu digo a mim mesmo o quanto já consegui resolver a situação", a maioria dos estudantes respondeu "indiferente" quanto ao uso da automotivação, afirmando a si mesmo o quanto já conseguiu realizar algo. Já nos itens "Quando passo por algum problema na universidade, encaro a situação por etapas, fazendo uma coisa de cada vez", "Eu tento evitar que os meus sentimentos atrapalhem em outras coisas na minha vida" e "Quando passo por algum problema, busco encontrar diferentes soluções", todos eles referentes ao coping focado no problema, a maioria das respostas foi em "concordo parcialmente". Carlotto (2013) diz que esse tipo de estratégia costuma atuar na origem do estresse como uma forma de confronto, objetivando a resolução do problema.

Os itens referentes ao coping focado na emoção, "Quando passo por um problema, procuro um culpado para a situação", "Quando me vejo diante de um problema, procuro me afastar das pessoas em geral", e "Eu culpo as pessoas que me causaram algum problema na universidade", tiveram maior número de respostas na opção "discordo totalmente". Na questão que afirma o desejo de mudar a forma como o indivíduo se sente em relação aos problemas da universidade, a maioria dos estudantes afirmou concordar totalmente. Carlotto (2013) afirma que os indivíduos que utilizam esse tipo de estratégia para enfrentar seus problemas costumam utilizar seus esforços para regular o estado emocional, e acabam deixando de lado a tentativa de solucionar os problemas.

Por fim, os últimos itens da pesquisa, que se referem às estratégias de enfrentamento focalizado na busca de suporte social, tiveram sua maior quantidade de respostas nas afirmações concordo parcialmente e totalmente. Os itens foram: "Quando enfrento um problema, converso com alguém sobre como estou me sentindo", "Quando me vejo diante de um problema, peço conselho a um parente ou um amigo que eu respeite", e "Quando passo por algum problema, tento guardar meus sentimentos para mim mesmo".

Nessas questões, foi abordada a busca por soluções dos problemas através das relações interpessoais, como família e amigos. Entretanto um fator que chamou atenção foi que, ao mesmo tempo em que os estudantes responderam buscar resolver os problemas por meio de relações interpessoais, eles dizem que guardam seus sentimentos para si mesmo, como é percebido no item "Quando passo por algum problema, tento guardar meus sentimentos para mim mesmo", mostrando assim certa contradição em suas afirmações.

Através dos resultados apresentados, pode-se perceber que a maioria dos estudantes costuma utilizar como mecanismo de enfrentamento as estratégias focalizadas no problema e na busca por suporte social. Zonta, Robles e Grosseman (2006) em seu estudo com estudantes de medicina, descrevem a valorização dos relacionamentos interpessoais e o trabalhar com a própria personalidade como meios eficazes para promover a qualidade de vida do estudante.

\section{Considerações Finais}

Com base nos dados coletados na pesquisa, percebe-se que os estudantes de administração do CAA/UFPE reconhecem que enfrentam diversas dificuldades no seu cotidiano, tais como sentimento de cansaço o tempo todo (77\%), insatisfação ao realizar as atividades diárias $(74,6 \%)$, mudança de humor constante $(61,7 \%)$, dificuldade de lembrar-se das coisas $(61,7 \%)$, tristeza $(59 \%)$, insônia e alteração significativa de sono (57,4\%), dificuldade de tomar decisões (54,7\%), dificuldade de pensar com clareza $(48,8 \%)$, dores de cabeça $(41 \%)$, choro constante $(28,5 \%)$, falta de apetite $(27 \%)$.

Podemos chamar essas dificuldades de sintomas, e a união de diversos sintomas pode ser considerada uma síndrome que, quando classificada pelo DSM-5 e pelo CID-10, recebe o nome de transtorno mental. Ainda em relação aos sintomas, foi possível perceber que as mulheres foram maioria para aqueles que responderam sim às questões sobre os sintomas de transtornos comuns, indicando um maior índice de desenvolvimentos desses transtornos nos estudantes do gênero feminino.

Dentre os fatores que podem influenciar no desenvolvimento dos sintomas mencionados pelos estudantes, merece destaque o nervosismo e a preocupação gerada no estudante antes da 
realização de alguma atividade acadêmica, uma vez que este fator foi considerado como principal problema por $43,8 \%$ dos estudantes. Em seguida apareceu que o nervosismo, tensão e a preocupação já foram motivos que levaram os estudantes a terem um baixo desempenho acadêmico com $38,3 \%$, seguindo pela cobrança feita a si mesmo para ter um ótimo desempenho acadêmico $(34,8 \%)$ e dificuldade de se perdoar quando obtém um baixo desempenho na academia $(28,5 \%)$. Um fator que chamou atenção foi a insatisfação dos estudantes com o conteúdo visto de sala de aula, uma vez que $89(34,8 \%)$ considera o conteúdo pouco aproveitável no mercado de trabalho.

Como mecanismo de enfrentamento dos estudantes, os instrumentos de defesa mais utilizados para lidar com os transtornos são aqueles focalizados no problema e no suporte social, seja de amigos ou familiares. Diante disso, conclui-se que boa parte dos estudantes investigados sofre de Transtornos Mentais Comuns (TMC), termo que classifica a existência de sintomas considerados inespecíficos, os quais não são classificáveis nos diagnósticos médicos e psiquiátricos. Além disso, percebeu-se ainda a possibilidade do desenvolvimento de Transtorno Depressivo Maior (TDM) em 13 estudantes, o que equivale a 5,1\% do total de entrevistados, uma vez que eles responderam de modo afirmativo a todos os 11 sintomas considerados nessa pesquisa.

Como sugestões de pesquisas futuras, propõem-se uma investigação qualitativa sobre os motivos pelos quais sintomas dos transtornos mentais comuns se apresentam mais em mulheres, uma pesquisa envolvendo exclusivamente os professores, como também a realização de estudos relacionados à realidade dos estudantes que moram longe dos pais, ou até mesmo daqueles que viajam todos os dias para estudar.

\section{Referências}

Almeida, S. L. \& Ferreira, J. A. (1997). Questionário de Vivências Académicas. Braga: Universidade do Minho.

Almondes, K. M., \& Araujo, J. F. (2003). Padrão do ciclo sono-vigília e sua relação com a ansiedade em estudantes universitários. Estudos de Psicologia [online], 8(1) 37-43. http://dx.doi.org/10.1590/S1413-294X2003000100005.

American Psychiatric Association. (2014). Manual diagnóstico e estatístico de transtornos mentais: DSM-5. Porto Alegre: Artmed.

Andrade, Antonio dos Santos, Tiraboschi, Gabriel Arantes, Antunes, Natália Amaral, Viana, Paulo Vinícius Bachette Alves, Zanoto, Pedro Alves, \& Curilla, Rafael Trebi. (2016). Vivências Acadêmicas e Sofrimento Psíquico de Estudantes de Psicologia. Psicologia: Ciência e Profissão, 36(4), 831-846. https://dx.doi.org/10.1590/1982-3703004142015

Andrade, João Brainer Clares de, Sampaio, José Jackson Coelho, Farias, Lara Maciel de, Melo, Lucas da Ponte, Sousa, Dalmy Pinheiro de, Mendonça, Ana Luisa Barbosa de, Moura Filho, Francisco Felinto Aguiar de, \& Cidrão, Ingrid Sorensen Marinho. (2014). Contexto de formação e sofrimento psíquico de estudantes de medicina. Revista Brasileira de Educação Médica, 38(2), 231-242. https://dx.doi.org/10.1590/S0100-55022014000200010.

Andrade, A. G., Duarte, P. C. A. V., \& Oliveira, L. G. (2010). (Orgs.) I Levantamento Nacional sobre o Uso de Álcool, Tabaco e Outras Drogas entre Universitários das 27 Capitais Brasileiras. Brasília: Secretaria Nacional de Políticas sobre Drogas (SENAD).

Assis, A. D. (2010). Vida universitária e Saúde Mental: Atendimento às demandas de saúde e Saúde Mental de estudantes de uma universidade brasileira. Cadernos Brasileiros de Saúde Mental, 2(4-5), 159-177.

Alves, T. (2014). Depressão e ansiedade entre estudantes da área de saúde. Revista De Medicina, 93(3), 101-105. https://doi.org/10.11606/issn.1679-9836.v93i3p101-105.

Abrao, Carolina Borges; Coelho, Ediane Palma; Passos, Liliane Barbosa da Silva. (2008). Prevalência de sintomas depressivos entre estudantes de medicina da Universidade Federal de Uberlândia. Rev. bras. educ. med., Rio de Janeiro, 32(3), 315-323. 
Aquino, Daniele Ramos de, Cardoso, Rodrigo Alves, \& Pinho, Lucinéia de. (2019). Sintomas de depressão em universitários de medicina. Boletim - Academia Paulista de Psicologia, 39(96), 81-95.

Barreto, I. S., Bezerra, A. L. Q. \& Barbosa, M. A. (2005). Assistência universitária - compromisso social. Revista da UFG, 7(2), dezembro. https://doi.org/10.5216/revufg.v7i2.48785.

Cerchiari, E. A. N. (2004). Saúde mental e qualidade de vida em estudantes universitários. Tese (Doutorado em Ciências Médicas, Universidade Estadual de Campinas). Campinas.

Castro, A. K. S. S., \& Teixeira, M. A. P. (2014). Evasão universitária: modelos teóricos internacionais e o panorama das pesquisas no Brasil. Psicologia Argumento, 32(2).

Cartollo, R. C. (2013). Adaptação Acadêmica e Coping em Estudantes Universitários. Dissertação (Mestrado em Psicologia, Centro de Ciências Sociais e Humanas, Universidade Federal de Santa Maria) Rio Grande do Sul.

Carlesso, J. (2020). Os desafios da vida acadêmica e o sofrimento psíquico dos estudantes universitários. Research, Society and Development, 9(2), e82922092. doi:http://dx.doi.org/10.33448/rsd-v9i2.2092.

Costa, E. S., \& Leal, I. P. (2008). Um olhar sobre a saúde psicológica dos estudantes do ensino superior: Avaliar para intervir. In: Congresso Nacional de Psicologia da Saúde, 7; Anais[...]. Porto: Universidade do Porto. Disponível em: http://www.isabelleal.com/Portals/1/PDFs/7congresso/vii-congresso-saude-pp-213-216.pdf. Acesso em: $07 \mathrm{de}$ março de 2008.

Creswell, J. W. (2010). Projeto de pesquisa: métodos qualitativo, quantitativo e misto. 3a edição. Porto Alegre: Artmed/Bookman.

Cunha, M. A. B., Neves, A. A. de F., Moreira, M. E., Hehn, F. J., Lopes, T. P., Ribeiro, C. C. F., \& Watanabe, Á. de P. F. (2009). Transtornos psiquiátricos menores e procura por cuidados em estudantes de Medicina. Revista Brasileira de Educação Médica, 33(3), 321-328. https://doi.org/10.1590/S0100-55022009000300002.

Farias, R.V., \& Almeida, L. S. (2020). Expectativas académicas no Ensino Superior: Uma revisão sistemática de literatura. Revista E-Psi, 9(1), 68-93.

Ferraz, M. F., \& Pereira, A. S. (2002). A dinâmica da personalidade e o homesickness (saudades de casa) dos jovens estudantes universitários. Psicologia, Saúde \& Doenças [online], 3(2), 149164.

Fiorotti, K. P., Rossoni, R. R., Borges, L. H., \& Miranda, A. E. (2010). Transtornos mentais comuns entre os estudantes do curso de medicina: prevalência e fatores associados. Jornal Brasileiro de Psiquiatria, 59(1), 17-23. http://dx.doi.org/10.1590/S0047-20852010000100003.

FONAPRACE/ANDIFES. (2016). IV Pesquisa do perfil socioeconômico e cultural dos estudantes de graduação das Instituições Federais de Ensino Superior Brasileiras 2014. Uberlândia: FONAPRACE/ANDIFES. Disponível em: <http://www.andifes.org.br/categoria/documentos/biblioteca/publicacoes-andifes/>. Acesso em 04 mar. 2018.

Fonseca, M. L. G., Guimarães, M. B. L., \& Vasconcelos, E. M. (2008). Sofrimento difuso e transtornos mentais comuns: uma revisão bibliográfica. Revista de APS, 11(3), 285-294.

Furtado E. S., Falcone, E. M. O. \& Clark, C. (2003). Avaliação do estresse e das habilidades sociais na experiência acadêmica de estudantes de medicina de uma universidade do Rio de Janeiro. Interação em Psicologia, 7(2), 43-51.

Giglio, J. S. (1976). Bem-estar emocional em estudantes universitários: Um estudo preliminar. Tese (Doutorado em Psicologia Médica e Psiquiátrica. Universidade Estadual de Campinas, Faculdade de Ciências Médicas). São Paulo, Campinas.

Leão, A.M., Gomes, I. P., Ferreira, M. J. M., \& Cavalcanti, L. P. G. (2018). Prevalência e Fatores Associados à Depressão e Ansiedade entre Estudantes Universitários da Área da Saúde de um Grande Centro Urbano do Nordeste do Brasil. Revista Brasileira de Educação Médica, 42(4), 55-65. https://doi.org/10.1590/1981-52712015v42n4rb20180092 
Muller, M. R., \& Guimaraes, S. S. (2007). Impacto dos transtornos do sono sobre o funcionamento diário e a qualidade de vida. Estudos de psicologia (Campinas), 24(4), p.519-528. http://dx.doi.org/10.1590/S0103-166X2007000400011

Nogueira, Maria José, Barros, Luísa, \& Sequeira, Carlos. (2017). A Saúde Mental em Estudantes do Ensino Superior: Relação com o género, nível socioeconómico e os comportamentos de saúde. Revista Portuguesa de Enfermagem de Saúde Mental, (spe5), 51-56.

OMS. (2002). Relatório Mundial da Saúde. Saúde mental: nova concepção, nova esperança. Lisboa: Ministério da Saúde Direcção-Geral da Saúde.

Silva P. L. B. C., Silva B. F. F., Chagas K. K. A. C. R., Tortola M. B. A., \& Caldeira L.R.L.(2019). Transtorno Mental Comum entre Estudantes de Enfermagem e FatoresEnvolvidos. Rev Enferm Cent-Oeste Min., 9:e3191.

Seidl, E. M. F., Troccoli, B. T., \& Zannon, C. M. L. C. (2001). Análise fatorial de uma medida de estratégias de enfrentamento. Psicologia: Teoria e Pesquisa, 17(3), 225-234. http://dx.doi.org/10.1590/S0102-37722001000300004

Victoria, M. S., Bravo, A., Felix, A. K., Neves, B. G., Rodrigues, C. B., Ribeiro, C. C. P., Canejo, D., Coelho, D., Sampaio, D., Esteves, I. M., Silva, J. A., Marotta, L., Rosa, M. S., Ribeiro, M. Y., Santos, N. S., Barbosa, T. B., Silva, T. M., Brito, T. M., Santos, V. C., Lima, V., \& Saltoris, W. P. (2013). Níveis de Ansiedade e Depressão em Graduandos da Universidade do Estado do Rio de Janeiro (UERJ). Encontro Revista de Psicologia, 16 (25) 163-165. https://revista.pgsskroton.com/index.php/renc/article/view/2447

Xavier, A., Nunes, A. I. B. L., \& Santos, M. S. (2008). Subjetividade e sofrimento psíquico na formação do Sujeito na Universidade. Revista Mal-estar e Subjetividade, VIII (2), 427-451.

Zonta, R., Robles, A. C. C. \& Grosseman, S. (2006). Estratégias de enfrentamento do estresse desenvolvidas por estudantes de medicina da Universidade Federal de Santa Catarina. Revista Brasileira de Educação Médica, 30, 147-153.

\title{
Common Mental Disorders in Business Students at a Public University in Agreste of Pernambuco
}

\begin{abstract}
This paper, entitled Common Mental Disorders in Business Administration Students at a Public University, aims to analyze what are the common mental disorders among Business Administration students at the Academic Center of Agreste, Federal University of Pernambuco. To this end, a quantitative, exploratory and descriptive research was conducted with 256 students. Sociodemographic identification of the subjects, mapping of common mental disorders, factors that contribute to the disorders, and coping mechanisms used were performed. Among the results found, there is a higher index of symptoms in female students, motivating factors associated with nervousness and self-esteem, as well as the predominant use of defense tools focused on the problem and social support.
\end{abstract}

Keywords: Common Mental Disorder; Mental health; Administration course. 


\section{Sobre os Autores}

\section{Rayssa Arruda MELO}

Bacharela em Administração. Universidade Federal de Pernambuco (UFPE), Centro Acadêmico do Agreste (CAA). Av. Campina Grande, s/n, Nova Caruaru, Caruaru, PE, Brasil, CEP 55.014900.

E-mail: ray.arruda@outlook.com

\section{Elisabeth Cavalcante dos SANTOS}

Doutora em Administração. Docente permanente Programa de Pós-Graduação em Gestão, Inovação e Consumo (PPGIC), Universidade Federal de Pernambuco (UFPE), Centro Acadêmico do Agreste (CAA). Av. Campina Grande, s/n, Nova Caruaru, Caruaru, PE, Brasil, CEP 55.014900.

E-mail: elisabethcsantos@gmail.com

\section{Pâmela Karolina DIAS}

Mestranda em Administração. Programa de Pós-Graduação em Gestão, Inovação e Consumo (PPGIC), Universidade Federal de Pernambuco (UFPE), Centro Acadêmico do Agreste (CAA). Av. Campina Grande, s/n, Nova Caruaru, Caruaru, PE, Brasil, CEP 55.014-900.

E-mail: pamela_dias2009@hotmail.com 